\title{
Differential recall of typical and atypical sentences as a function of retrieval cue
}

\author{
LORRAINE A. LOW, CHARLES A. ROSSIGNOL, AUDREY ELMONT MIRLOCCA, \\ and MAUREEN TYRRELL \\ Framingham State College, Framingham Center, Massachusetts 01701
}

\begin{abstract}
The present study was designed to determine the differential retrieval of typical and atypical sentences given subject, object, or inference cues. Typical sentences were ones in which the predicate expressed an action that was characteristic of the role definition of the subject. Atypical sentences contained predicates that expressed actions not confined to this role definition. Third-graders, sixth-graders, and freshman college students were given lists of typical and atypical sentences followed by a subject, object, or inference cue as the appropriate retrieval cue for recall of the entire sentence. Subject cues were found to be the best retrieval cues and also differentiated the recall of typical and atypical sentences. Atypical sentences, given the subjects as retrieval cues, resulted in better recall than did typical sentences. The findings were interpreted as reflecting the unique encoding of sentences that are atypical.
\end{abstract}

Rosenberg (1968, 1969) has demonstrated that semantically well-integrated (SWI) sentences are better recalled than semantically poorly integrated (SPI) sentences. The degree of semantic integration was defined by empirically determined norms in which the subject of the senterice was provided and the verb and object were associatively provided. The sentences "The doctor cured the patient" and "The doctor shook the author" are SWI and SPI sentences, respectively.

The explanation for the superior recall of SWI sentences was the greater chunking or more holistic storage of this material compared with the SPI sentences. According to Horowitz and Prytulak (1969), the better retention of more holistic material can be attributed to two separate factors. The first is the superior recall of a portion of the sentence, and the second is the ability of the recalled portion to redintegrate the remaining portions of the sentence. It is possible, then, that the superiority of the SWI sentences is attributable to better initial recall, to better redintegration of the remainder once a portion has been recalled, or to both factors.

Alternatively, one can argue that once a portion of the sentence is recalled, SPI sentences should be more easily redintegrated than SWI sentences. The lack of familiarity with SPI sentences might lead to better attending and hence more specific encoding. Indeed, Schnur (1977) has demonstrated that atypical members of categories are recalled better than typical members.

The present experiment was designed to determine the relative ability of retrieval cues in SWI and SPI sentences in redintegrating the complete sentence. In the present experiment, sentences were constructed in such a manner that the subject noun had a definitive role (e.g., pilot, babysitter, plumber). The SWI sentences were here defined as typical, since the predicate expressed an action typical of the role. Similarly, atypical sentences were chosen so that the action was not expected from the particular role definition. Differences between the two types were accomplished by substituting verbs as follows: "The shoemaker pounded the boots" (typical). "The shoemaker wore the boots" (atypical). For recall, either the subject or object of the sentence or an inference cue (one that instantiated the action of the sentence) provided the single retrieval cue for that sentence.

It was expected that differences in retrieval of these two types would also be age related, since prior experience is a crucial determinant of the degree to which a sentence is given holistic meaning.

\section{METHOD}

\section{Subjects}

The subjects were third-graders, sixth-graders, and college freshmen, 20 from each group. The children were randomly selected from a population in local elementary schools. The college students were volunteer freshmen from Framingham State College.

\section{Stimulus Material and Design}

Two lists, A and B, were constructed, with 36 sentences in each list. Each sentence contained only one subject, one verb, and one object. A typical sentence in one list was atypical in the second list. This was accomplished by replacing the verb and holding constant the remainder of the sentence. Thus, "The thief grabbed the diamonds" (typical) became "The thief wore the diamonds" (atypical) in the second list. Each block of six sentences contained all combinations of the two types of sentences (typical and atypical) and three cue types (subject, object, and inference), with only one cue type assigned to each sentence. Each age group was randomly divided into two groups, one receiving List $A$ and the other, List $B$. The design was a 3 (age) by 2 (list) by 2 (sentence typicality) by 3 (cue type) mixed factorial.

\section{Procedure}

The subject was instructed to try to remember each sentence as it was read to him, since his task was to recall as many sentences as possible. The sentences in each block were read, followed by presentation of the retrieval cues. Each retrieval cue 
was read to the subject, and he was instructed to recall part of the sentence if he could not recall the entire sentence.

\section{RESULTS}

The total number of sentences in which the subject, verb, and object were recalled was the response measure. An analysis of variance was performed on the data. Age differences were statistically significant $[\mathrm{F}(2,54)=27.22$, $\mathrm{p}<.01]$. The means for third-graders, sixth-graders, and college freshmen were $2.4,2.0$, and 3.4 , respectively, indicating that the college freshmen performed better than either third-graders or sixth-graders. Differences associated with sentence typicality were also statistically significant $[F(1,108)=14.16, p<.01]$. The means for typical and atypical sentences were 2.43 and 2.78 , respectively, indicating that atypical sentences were recalled significantly more than typical sentences. The effect of cues was also statistically significant $[F(2,108)=53.86, p<.01]$. The means of $3.2,2.8$, and 1.8 for subject, object, and inference cue, respectively, indicated that the subject was the most effective cue and the object was the least effective cue for sentence retrieval. The Newman-Keuls test for specific comparisons of score data indicated that all the above pairwise comparisons were statistically significant. Age by Cue Type was statistically significant $[F(2,108)=4.57$, $\mathrm{p}<.05]$. Contrary to prediction, Age by Sentence Typicality was not statistically significant. The inference cue was the only cue linearly and positively related to age, with means of $.8,1.8$, and 2.8 for third-graders, sixth-graders, and college students, respectively. Sentence Typicality by Cue Type was statistically significant $[F(2,108)=4.57, p<.05]$. The means of the typical sentences were $2.8,2.7$, and 1.8 for subject, object, and inference cue, respectively, and for atypical sentences they were 3.6, 2.8, and 1.9 for subject, object, and inference cues, respectively. A Tukey specificcomparisons test for unconfounded means in an interaction table indicated that significant differences between typical and atypical sentences were significant only with the subject as retrieval cue $(\mathrm{p}<.01)$. Atypical sentences were therefore better recalled than typical sentences only when the subject was the retrieval cue.

\section{DISCUSSION}

The present finding that the subject is the best retrieval cue is consistent with other studies (Horowitz \& Prytulak, 1969; Thios, 1975). The important finding of the present study is that atypical sentences are better recalled than typical sentences when the subject of the sentence is the retrieval cue. These findings are not inconsistent with those of Rosenberg (1968, 1969) if we assume that in a free recall situation the probability of eliciting part of the typical sentence is higher than that for an atypical sentence. Once the subject is provided with the retrieval cue, however, the remainder of the atypical sentence is recalled with a higher probability than the typical sentence.

We can account for the present findings if we assume that the atypical sentence is more uniquely encoded. Schnur's (1977) finding that low-ranking prototypical exemplars are recalled better than high-ranking exemplars was explained in that study by the high information content provided by the former relative to the latter. Thus, the relative unfamiliarity of the atypical sentences in the present experiment could have similarly led to this more specific encoding.

An alternative explanation for the present findings is provided by the nature of the particular sentences in the present study. The difference between the two sentence types was determined by verb change. Since changes in the verb produce change in the action, corresponding differences in imagery level could have easily been produced. Thus atypical sentences might have been associated with higher imagery levels than typical sentences. An added control in a future study might be change in the subject noun with constant verb and object.

The failure of sentence typicality to interact with age might reflect the simplicity of the sentences used. The negative results could be interpreted as indicating that even at the third-grade level, typical and atypical sentences are encoded differently.

\section{REFERENCES}

Horowitz, L. M., \& Prytulak, L. S. Redintegrative memory. Psychological Review, 1969, 76, 519-531.

Rosenberg, S. Association and phrase structure in sentence recall. Journal of Verbal Learning and Verbal Behavior, 1968, 7, 1077-1081.

ROSENBERG, S. The recall of verbal material accompanying semantically well-integrated and semantically poorly-integrated sentences. Journal of Verbal Learning and Verbal Behavior, $1969,8,732-736$.

SchnuR, P. Testing the encoding elaboration hypotheses: The effects of exemplar ranking on recognition and recall. Memory \& Cognition, 1977, 5, 666-672.

Thios, S. J. Memory for general and specific sentences. Memory \& Cognition, 1975, 3, 75-77.

(Received for publication April 1, 1980.) 